\title{
TWO $R$-CLOSED SPACES REVISITED
}

\author{
STEPHEN H. HECHLER 1
}

\begin{abstract}
Recently, R. M. Stephenson has used the Continuum Hypothesis to construct two $R$-closed, separable regular, first countable, noncompact Hausdorff spaces. We show that the assumption of the Continuum Hypothesis can be removed by replacing a lemma used in the original construction to deal with arbitrary almost-disjoint families by the construction of a particular almost-disjoint family. We also show that while these spaces always have cardinality $c$, it is at least consistent with the negation of the Continuum Hypothesis that there exist spaces with the same properties, but which have cardinality $\kappa_{1}$. We conclude with some consistency results concerning relationships between open filter bases and generalizations of the notions of feeble compactness and Lindelöfness.
\end{abstract}

In $[8, \S 4]$ R. M. Stephenson constructs two spaces $(S, \mathcal{S})$ and $(T, \mathcal{T})$ which are both separable, first countable, feebly compact, not countably compact, regular, and Hausdorff. Further, $(S, \delta)$ is not minimal regular, but if the Continuum Hypothesis holds, then $(S, \delta)$ is $R$-closed and $(T, \mathcal{T})$ is strongly minimal regular. We shall prove that the construction may be modified in such a way as to insure that the spaces retain these latter properties without the assumption of any special set theoretical hypotheses (other than the Axiom of Choice which we shall assume throughout and without further mention), and we shall prove that it is consistent with the negation of the Continuum Hypothesis that these spaces have cardinality $\boldsymbol{\aleph}_{1}$. These spaces are of importance because they appear to be the only known examples of separable first countable $R$-closed spaces which are not compact. We shall also consider consistency results concerning generalizations of the notions of feeble compactness and Lindelöfness. These will give us, for example, conditions under which every open filter over a space has an adherent point.

Although many of our results will be topological in nature, our constructions will be set theoretical and will be self-contained. However, because Stephenson's original construction, while very clever, is quite involved, we shall not repeat it here, and a detailed knowledge of [8] would be required to reconstruct the two $R$-closed spaces referred to in the title. In what follows, all spaces are assumed to be at least $T_{1}$.

In Stephenson's construction, the Continuum Hypothesis is used to couple two lemmas. One (Lemma 4.1) states that in a certain space there is a subset

Received by the editors November 18, 1974 and, in revised form, March 3, 1975 and August 7, 1975.

AMS (MOS) subject classifications (1970). Primary 54A25, 54D25; Secondary 02K25, 04A30.

${ }^{1}$ The preparation of this paper was supported by a grant from the City University of New York Faculty Research Program. 
$A$ of cardinality c such that if any open set contains c points of $A$, its closure contains c points of the complement of $A$. Then in $(S, \mathscr{S})$ and $(T, \mathcal{T})$ if one has a regular filter base such that the closure of each member has cardinality c, this may be used to prove that the "extreme points" are adherent points. On the other hand, another lemma (Lemma 4.3) states that if the closure of some member of an open filter base in these spaces is countable, then again the filter base has an adherent point (though not necessarily one of the extreme points). Thus if there are no cardinals between $\aleph_{0}$ and $\mathbf{c}$, all cases are covered, and every open filter base must have an adherent point. We shall show that if we choose a particular member of the class of spaces to which Lemma 4.1 is applied, then even if the Continuum Hypothesis fails, we may replace $\mathrm{c}$ by $\boldsymbol{\kappa}_{1}$ in the statement of the lemma. Hence if we use this particular space as the building block for the final construction, all cases will again be covered.

We shall need some notation. Let $N$ denote the set of natural numbers, let $Q$ denote the rationals, and let $R$ denote the reals. For any set $A$ let $\mathcal{P}(A)$ denote the power set of $A$, and let $\mathcal{P}^{*}(A)$ denote the set of infinite subsets of $A$. For $A$ and $B$ denumerable, define

$$
A \subseteq^{*} B \text { iff } A-B \text { is finite, and } A=^{*} B \text { iff } A \subseteq^{*} B \text { and } B \subseteq^{*} A .
$$

Then define $A$ to be almost contained in $B$ iff $A \subseteq^{*} B$, define $A$ and $B$ to be almost disjoint iff their intersection is finite, and define an infinite set $\Re \subset \mathscr{P}^{*}(A)$ to be a (maximal) almost-disjoint family over $A$ iff its elements are pairwise almost disjoint (and it is not properly included in any other almostdisjoint family over $A$ ). We shall abbreviate by referring to a maximal almostdisjoint family as a MADF or a $\kappa$-MADF if of cardinality $\kappa$.

We now consider the class of spaces used by Stephenson (which he credits to $\mathrm{J}$. Isbell and $\mathrm{S}$. Mrówka). As we shall need to examine the construction, we repeat it here. Let $\Re$ be any MADF over $N$, and let $D=\left\{p_{M}: M \in \Re\right\}$ be any new set of distinct points. Then $B_{\Re}$ is defined to be the space whose set of points is $N \cup D$ with the topology generated by letting each point of $N$ be isolated and by letting a neighborhood of a point $p_{M}$ be any set containing $p_{M}$ and all but finitely many members of $M$. Stephenson proves for an arbitrary c-MADF $\Re$ that:

Lemma 4.1 (STEPHenson). There exists a subset $A$ of $D$ such that $|A|=\mathrm{c}$ and for every open subset $U$ of $B_{\Re}$

$$
|U \cap A|=\mathbf{c} \rightarrow|\bar{U} \cap(D-A)|=\mathbf{c} .
$$

A study of the construction used shows us that to obtain the desired properties in the final spaces without recourse to the Continuum Hypothesis, it would be sufficient to prove that in this lemma (1) can be replaced by

$$
|U \cap A|>\aleph_{0} \rightarrow|\bar{U} \cap(D-A)|>\aleph_{0} .
$$

While we do not know if this replacement can always be made, we shall construct a particular family for which it can be, and we shall prove that this new family has even stronger properties. To do this it will be necessary to translate Lemma 4.1 with (1) replaced by (2) into a statement concerning $\Re$. It is not hard to see that this statement becomes 
Lemma 4.1*. There exists a subfamily $\mathscr{Q} \subset \mathfrak{\Re}$ of cardinality $\mathbf{c}$ such that for every set $U \in \mathscr{P}^{*}(N)$

$$
\begin{aligned}
\mid\{A \in \mathbb{Q}: & \left.A \cap U \neq^{*} \varnothing\right\} \mid>\aleph_{0} \\
\rightarrow & \left|\left\{A \in(\Re-\mathscr{Q}): A \cap U \neq^{*} \varnothing\right\}\right|>\aleph_{0} .
\end{aligned}
$$

Before carrying out our construction it will be convenient to prove a variant of the well-known theorem [2] which states that there exists a set of reals such that neither it nor its complement includes a perfect set.

Lemma 1. There exists a set $S \subset R$ such that if $F$ is any uncountable closed subset of $R$, then $|F \cap S|=|F-S|=\mathbf{c}$.

Proof. Since there are exactly $\mathrm{c}$ uncountable closed subsets of $R$, let $\left\{F_{\alpha}: \alpha<\mathbf{c}\right\}$ be any listing of these sets such that each appears in the list $\mathbf{c}$ times. Then, as usual, construct $S$ inductively. That is, at stage $\alpha$ choose two points in $F_{\alpha}$ which have not yet been used and put one in $S$ and the other in the complement of $S$. We leave the details to the reader.

We now construct $\mathscr{F}$, and in order to make use of the topology of $R$, we construct it over $Q$ rather than $N$. Thus for each real number $r \in R$ let $\mathcal{P}_{r}^{*}(Q)$ be the set of bounded subsets $A$ of $Q$ such that the derived set $A^{\prime}$ of $A$ with respect to $R$ (the set of points in $R$ which are accumulation points of $A$ ) contains the single point $r$. In fact, such a subset is simply the range of a sequence converging to $r$. Now, for each real number $r$ let $\mathscr{F}_{r}$ be any fixed almost-disjoint subfamily of $\mathscr{P}_{r}^{*}(Q)$ which is maximal with respect to $\mathscr{P}_{r}^{*}(Q)$, i.e. which is not properly included in any other such almost-disjoint family. It is easily seen that $r \neq s$ implies that if $F \in \mathscr{P}_{r}^{*}(Q)$ and $G \in \mathscr{P}_{s}^{*}(Q)$, then $F$ and $G$ are almost disjoint. Hence the family $\cup\left\{\mathscr{F}_{r}: r \in R\right\}$ is almost disjoint, and we may choose a maximal almost-disjoint family $\mathscr{F}$ over $Q$ which includes it.

Using this family, we may replace Lemma $4.1^{*}$ by

THEOREM 1. There exists a subfamily $\mathbb{C} \subset \mathscr{F}$ of cardinality $\mathrm{c}$ such that for every set $U \in \mathcal{P}^{*}(Q)$

(4) $\left|\left\{A \in \mathbb{Q}: A \cap U \neq{ }^{*} \varnothing\right\}\right|>\aleph_{0} \rightarrow\left|\left\{A \in(\mathscr{F}-\mathscr{Q}): A \cap U \neq{ }^{*} \varnothing\right\}\right|=\mathbf{c}$.

Proof. Let $S$ be the set from Lemma 1 . Then for each $s \in S$ choose a fixed set $F_{s} \in \mathscr{F}_{s}$, and let $\mathbb{Q}=\left\{F_{s}: s \in S\right\}$.

Now let $U$ be any subset of $Q$, and let $U^{\prime}$ again be the derived set of $U$ in $R$. It follows immediately from the construction that

$$
r \in U^{\prime} \leftrightarrow \exists F \in \mathscr{F}_{r}\left(U \cap F \neq{ }^{*} \varnothing\right) .
$$

Thus if the set $\left\{A \in \mathbb{Q}: A \cap U \neq^{*} \varnothing\right\}$ is uncountable, there is an uncountable subset of $S$ contained in $U^{\prime}$. This then implies that $U^{\prime}$ is a closed uncountable subset of $R$, so there must exist a set $T \subseteq\left(U^{\prime}-S\right)$ of cardinality c. But for each $t \in T$ there must be at least one set $F_{t} \in \mathscr{F}_{t} \subset(\mathscr{F}-\mathscr{Q})$ such that $U \cap F_{t} \neq^{*} \varnothing$.

Since (4) clearly implies (3), Stephenson's Theorem 4.4 generalizes to

THEOREM 2. There exist two R-closed separable first-countable spaces $(S, \delta)$ 
and $(T, \mathfrak{T})$ such that $(S, \mathcal{S})$ is not minimal regular, $(T, \mathfrak{T})$ is strongly minimal regular, and neither is countable compact.

We next consider questions concerning the cardinalities of these spaces. Since each consists of a countable collection of copies of $B_{\mathscr{F}}$ suitably connected, those we have looked at have cardinality c. However, it is certainly consistent with the negation of the Continuum Hypothesis that there exist maximal almost-disjoint families of cardinality less than $\mathbf{c}$, and it is reasonable to believe that we might use these families or modifications of them to construct smaller spaces. Unfortunately, we do not have a version of Lemma 4.1 which requires only the existence of small families, so we shall be forced to consider several other set theoretical hypotheses.

In order to simplify the statements of several of our theorems, we define a $\kappa$-Stephenson pair to be a pair of spaces constructed from a $\kappa$-MADF as in [8] and which, therefore, have cardinality $\kappa$ and satisfy the conclusions of Theorem 2. Thus Theorem 2 states that there always exists a c-Stephenson pair. We next reduce the question of the existence of $\kappa$-Stephenson pairs to a combinatorial question by noting that the construction depends upon the existence of appropriate partitions of MADF's. Therefore, for any MADF $\pi$ we define a set $\mathscr{Q} \subset \Re$ of cardinality $|\Re-\mathscr{Q}|=|\Re|$ to be a $(\gamma, \delta)$-partition iff for every set $U \in \mathscr{P}^{*}(N)$

$$
\left|\left\{A \in \mathbb{Q}: A \cap U \neq^{*} \varnothing\right\}\right| \geqslant \gamma \rightarrow\left|\left\{A \in(\mathfrak{R}-\mathbb{Q}): A \cap U \neq^{*} \varnothing\right\}\right| \geqslant \delta .
$$

It then follows immediately from Stephenson's construction that

LEMMA 2. If for an infinite cardinal $\kappa$ there exists a $\kappa-M A D F$ which admits either an $\left(\aleph_{0}, \aleph_{0}\right)$-partition or an $\left(\aleph_{1}, \aleph_{1}\right)$-partition, then there exists a $\kappa$ Stephenson pair.

We now consider some consistency results. In [3] we constructed a model of Zermelo-Fraenkel set theory in which the Continuum Hypothesis failed, but in which for each uncountable cardinal $\kappa \leqslant c$ there existed a $\kappa$-MADF $\mathbb{Q}_{\kappa}$. We now note that by standard forcing techniques it can be seen that each such $\mathbb{Q}_{\kappa}$ admits an $\left(\boldsymbol{\kappa}_{0}, \kappa\right)$-partition. Since such a partition is necessarily an $\left(\boldsymbol{\aleph}_{0}, \boldsymbol{\kappa}_{0}\right)$ partition, we have

TheOREM 3. It is consistent with the negation of the Continuum Hypothesis that there exist a $\kappa$-Stephenson pair for each uncountable cardinal $\kappa \leqslant \mathbf{c}$.

While we cannot say any more in the general case, we do have some further results with respect to a specific cardinal $\mathbf{K}$ which we define to be the smallest infinite cardinal $\kappa$ for which there exists a $\kappa$-MADF. These will be based on the fact that

THEOREM 4. For every cardinal $\kappa \leqslant \mathbf{K}$ every $(\mathbf{K}, \kappa)$-partition of a MADF is also an $\left(\boldsymbol{\aleph}_{0}, \kappa\right)$-partition.

Proof. Let $\mathscr{Q}$ be a $(\mathbf{K}, \kappa)$-partition of a MADF $\Re$, and let $U \in \mathscr{P}^{*}(N)$. Now suppose that $\kappa_{0} \leqslant\left|\left\{A \in \mathbb{Q}: A \cap U \neq^{*} \varnothing\right\}\right|<\mathbf{K}$. Then the family $\{(B$ $\left.\cap U) \in \mathscr{P}^{*}(U): B \in \mathfrak{N}\right\}$ is a MADF over $U$ and, therefore, has cardinality at least $\mathbf{K}$. Hence the family $\left\{B \in(\Re-\mathscr{Q}): B \cap U \neq^{*} \varnothing\right\}$ also must have cardinality at least $\mathbf{K}$. 
COROLlary 1. It is consistent with the negation of the Continuum Hypothesis that every MADF be usable as the basis of a Stephenson pair.

Proof. It is known that Martin's Axiom [5] implies that $\mathbf{K}=\mathbf{c}$ and that it is consistent [7] with the negation of the Continuum Hypothesis. But Stephenson's Lemma 4.1 restated says that every c-MADF admits a (c, c)-partition.

COROLlARY 2. If there exists a K-MADF which admits a $(\mathbf{K}, 1)$-partition, then there exists a K-Stephenson pair.

Proof. It is easily seen that any $(K, 1)$-partition must also be a $\left(K, \boldsymbol{N}_{0}\right)$ partition, and this, by Theorem 4 , must be an $\left(\boldsymbol{N}_{0}, \boldsymbol{\aleph}_{0}\right)$-partition.

We do not know if these partitions always exist, but we can obtain some information by considering the

HYPOTHESIS $\mathbf{H}(\kappa, \lambda)$. For every family $\mathscr{F} \subseteq \mathcal{P}^{*}(\kappa)$ of cardinality $\lambda$ such that each member of $\mathcal{F}$ has cardinality $\kappa$ there exists a set $G \subset \kappa$ of cardinality $\kappa$ which does not contain any member of $\mathscr{F}$ as a subset.

It now follows that

THEOREM 5. H( $\kappa, \mathbf{c})$ implies that every $\kappa-M A D F$ admits $a(\kappa, 1)$-partition.

Proof. Let $\Re=\left\{M_{\alpha}: \alpha \in \kappa\right\}$ be any $\kappa$-MADF, and for each $S \in \mathcal{P}^{*}(N)$ let $F_{S}=\left\{\alpha \in \kappa: M_{\alpha} \cap S \neq^{*} \varnothing\right\}$. Then let $\mathscr{F}=\left\{F_{S}:\left|F_{S}\right|=\kappa\right\}$. Since $|\mathscr{F}|$ $\leqslant \mathbf{c}$, by hypothesis there exists a set $A \subset \kappa$ of cardinality $\kappa$ which admits no member of $\mathscr{F}$ as a subset. But from this it follows immediately that $\mathbb{Q}$ $=\left\{M_{\alpha}: \alpha \in A\right\}$ is a $(\kappa, 1)$-partition.

COROLlaRY 3. H(K, c) implies that every $\mathbf{K}-M A D F$ may be used to generate a K-Stephenson pair.

Unfortunately, we do not know very much about $\mathbf{H}(\kappa, \lambda)$, although it is a special case of some propositions considered by J. Baumgartner [1]. Thus we must consider the

HyPOTHESIS $\mathbf{B}(\kappa, \lambda)$. There exists a family $\mathscr{F} \subset \mathscr{P}(\kappa)$ of cardinality $\lambda$ such that each member of $\mathscr{F}$ has cardinality $\kappa$ but the intersection of any two distinct members of $\mathcal{F}$ has cardinality less then $\kappa$.

It is easily seen that $\mathbf{B}\left(\kappa, \lambda^{+}\right)$implies $\mathbf{H}(\kappa, \lambda)$. Baumgartner [1] has a series of interesting results concerning the hypotheses $\mathbf{B}(\kappa, \lambda)$; we mention one example here.

THeorem 6 (Baumgartner). If $\mathbf{c}$ is less than both $2^{\aleph_{1}}$ and $\aleph_{\omega}$, then $\mathbf{B}\left(\aleph_{1}, 2^{\aleph_{1}}\right)$ holds.

Combining this with our previous results yields

Corollary 4. If $\mathrm{c}$ is less than both $2^{\aleph_{1}}$ and $\aleph_{\omega}$, then every $\aleph_{1}-M A D F$ can be used to generate an $\aleph_{1}$-Stephenson pair. In particular, if there exists an $\aleph_{1}-M A D F$, then there exists an $\aleph_{1}$-Stephenson pair.

We conclude with a generalization of the following lemma in [8].

LeMMA 4.3 (STEPHENSON). If $\mathfrak{T}$ is any open filter base on a feebly compact 
space, then either it admits an adherent point or each of its members has uncountable closure.

For each infinite cardinal $\kappa$ define a space $T$ to be $\kappa$-Lindelöf iff every open cover of $T$ admits a subcover of cardinality at most $\kappa$ and to be $\kappa$-feebly compact iff every open filter base of cardinality at most $\kappa$ admits an adherent point. Now following Stephenson, we note that

LEMMA 3. Every open filter base on a $\kappa$-Lindelöf, $\kappa$-feebly compact space admits an adherent point.

We next consider the question of when a space is $\kappa$-feebly compact. Define a subset $S$ of a space $T$ to be $s$-dense (strongly dense) iff it is dense and every infinite subset of it admits an accumulation point in $T$, and define a space $T$ to be s-separable iff it admits a countable s-dense subset. Finally, for any infinite cardinal $\kappa$ consider the set theoretical

HYPOTHESIS $\mathbf{C}_{\kappa}$. If $\mathscr{F}$ is any family of subsets of $N$ such that $|\mathscr{F}| \leqslant \kappa$ and every finite intersection of members of $\mathscr{F}$ is infinite, then there is an infinite subset $F$ of $N$ which is almost contained in every member of $\mathscr{F}$.

Although $\mathbf{C}_{\boldsymbol{k}}$ does not appear to be topological in nature, we have

THEOREM 7. The hypothesis $\mathbf{C}_{\kappa}$ holds iff every s-separable $T_{1}$ space is $\kappa$-feebly compact.

Proof. Suppose $\mathbf{C}_{\boldsymbol{\kappa}}$ holds. Let $\mathfrak{V}$ be an open filter base of cardinality $\kappa$ on an $s$-separable $T_{1}$ space $T$, and let $S$ be a countable $s$-dense subset of $T$. Now let $\mathscr{F}=\{V \cap S: V \in \mathcal{V}\}$. Note that if any finite intersection of members of $\mathscr{F}$ is finite, then at least one member of the intersection will be contained in every member of $\mathfrak{V}$ and will, therefore, be an adherent point. If, however, every such intersection is infinite, then we may apply $\mathbf{C}_{\boldsymbol{K}}$ to obtain an infinite subset $F$ of $S$ which is almost contained in every member of $\widetilde{T}$. But because $S$ is $s$ dense, $F$ must admit an accumulation point, and because $T$ is $T_{1}$, this point must be an adherent point of $\mathcal{T}$.

In the other direction, suppose that every $s$-separable $T_{1}$ space is $\kappa$-feebly compact, and let $\mathscr{F}$ be a family of at most $\kappa$ subsets of $N$ such that every finite intersection of members of $\mathscr{F}$ is infinite. We first note that those members of $\mathscr{F}$ whose complements are finite cause no problems and may be disregarded. Similarly, if the set $\cap \mathscr{F}$ is infinite, it is the set we are looking for, but if it is finite, it may be deleted from $N$. Thus, without loss of generality, we may consider the case where $\cap \mathscr{F}$ is empty and the complement of every member of $\mathscr{F}$ is infinite. In this case let $D=\left\{p_{F}: F \in \mathscr{F}\right\}$ be a new set of points, and construct a topological space $T$ by setting $T=D \cup N$, letting points of $N$ be isolated, and defining neighborhoods of points $p_{F} \in D$ to be sets containing $p_{F}$ and all but finitely many points of $N-F$. It is easily seen that $T$ is a $T_{1}$ space and that $\mathscr{F}$ is an open filter base of cardinality at most $\kappa$ over $T$ which admits no adherent points. Thus $T$ is not $\kappa$-feebly compact and, therefore, is not, by hypothesis, $s$-separable. But $N$ is dense in $T$, and because it cannot be $s$-dense, there must exist an infinite subset $F$ of $N$ which admits no accumulation points in $T$. However, this implies that $F$ must be almost contained in every member of $\mathscr{F}$. 
Combining this with Lemma 3, we have

COROLlary 5. If $\mathbf{C}_{\kappa}$ holds and $T$ is any s-separable $T_{1}$ space, then:

(a) If $T$ is $\kappa$-Lindelöf, every open filter base on it has an adherent point.

(b) If there exists an open filter base $\mathfrak{V}$ on $T$ with no adherent points, then the closure of each member of $\mathfrak{V}$ has cardinality greater than $\kappa$.

(c) If $T$ is $\kappa$-Lindelöf and Hausdorff, then it is absolutely closed.

(d) If $T$ is $\kappa$-Lindelöf and regular, then it is compact.

Proof. Parts (b), (c) and (d) follow from part (a).

It can also be seen that by using Corollary 5(a) in one direction and modifying the proof of Theorem 7 slightly by noting that the space $T$ in the second part is $\kappa$-Lindelöf, we have

COROllary 6. The hypothesis $\mathbf{C}_{\kappa}$ is equivalent to the hypothesis that every open filter base over a $\kappa$-Lindelöf s-separable $T_{1}$ space admit an adherent point.

Since $\mathbf{C}_{\boldsymbol{\aleph}_{0}}$ always holds, and Martin's Axiom implies that $\mathbf{C}_{\boldsymbol{\kappa}}$ holds for all $\kappa<\mathbf{c}$ [5], we have

COROLlaRY 7. Every s-separable space is feebly compact, and it is consistent with the negation of the Continuum Hypothesis that every open filter base of cardinality strictly less than $\mathbf{c}$ over an s-separable space admit an adherent point.

The hypotheses $\mathbf{C}_{\kappa}$ have been studied before and are known to be quite strong. In particular, F. Rothberger [6] has shown that if $\mathbf{C}_{\kappa}$ holds, then $2^{\kappa}$ must equal $\mathbf{c}$, and the present author [4] has proven some results similar to Corollary 5. It is also well known that if $\mathcal{N}$ is any $\kappa$-MADF, then the family $\{N-M: M \in \mathfrak{N}\}$ is a counterexample to $\mathrm{C}_{\kappa}$. This latter is quite important because without it we would be tempted to look for a cardinal $\kappa$ such that there exists a $\kappa$-MADF with a $(\kappa, \kappa)$-partition and for which $\lambda<\kappa$ implies $\mathbf{C}_{\lambda}$. However, the only cardinal which could satisfy both these conditions is $\mathbf{K}$, and we have shown that in this case the $(\mathbf{K}, \mathbf{K})$-partition is sufficient by itself to construct the K-Stephenson pair.

We wish to thank the referee for his many useful comments.

\section{REFERENCES}

1. J. E. Baumgartner, Almost-disjoint sets, the dense-set problem, and the partition calculus (preprint).

2. F. Bernstein, Zur Theorie der trigonometrischen Reihen, Leipziger Berichte 60 (1908), 329.

3. S. H. Hechler, Short complete nested sequences in $\beta N-N$ and small maximal almost-disjoint families, General Topology and Appl. 2 (1972), 139-149. MR 46 \#028.

4. - On some weakly compact spaces and their products, General Topology and Appl. 5 (1975), 83-93.

5. D. A. Martin and R. M. Solovay, Internal Cohen extensions, Ann. Math. Logic 2 (1970), 143-178. MR 42 \# 5787.

6. F. Rothberger, On some problems of Hausdorff and of Sierpiniski, Fund. Math. 35 (1948), 29-46. MR 10, 689.

7. R. M. Solovay and S. Tennenbaum, Iterated Cohen extensions and Souslin's problem, Ann. of Math. (2) 94 (1971), 201-245. MR 45 \#3212.

8. R. M. Stephenson, Two R-closed spaces, Canad. J. Math. 24 (1972), 286-292. MR 45 \# 7665. 